\title{
Theoretical fertility McMullin-style
}

Forthcoming in the European Journal for the Philosophy of Science

A theory's fertility is one of the standard theoretical virtues. But how is it to be construed? In current philosophical discourse, particularly in the realism debate, theoretical fertility is usually understood in terms of novel success: a theory is fertile if it manages to make successful novel predictions. Another, more permissible, notion of fertility can be found in the work of Ernan McMullin. This kind of fertility, McMullin claims, gives us just as strong (or even stronger) grounds for realism. My paper critically assesses McMullin's notion of fertility and its realist rationale. It concludes that McMullin's preferred example, namely the fertile development of the Bohr-Sommerfeld model of the atom, does not support McMullin's argument for realism. Although the implications for the realism debate are as of yet unclear, the case study offers some important methodological lessons.

Keywords: theoretical fertility, theoretical virtue, novel success, realism, idealization, positive heuristics, Bohr-Sommerfeld model

\section{Introduction}

A theory's fertility, fruitfulness, or fecundity as first identified by T.S. Kuhn (1977) as one of the five most important virtues of theories. ${ }^{1}$ Indeed, although Kuhn regarded fertility as "somewhat less standard", he thought that fertility is "of special importance to actual scientific decisions". Curiously, he had not much to say about it, other than that it would amount to a theory "disclose[ing] new phenomena or previously unnoted relationships among those already known" (322). Nowadays, theoretical fertility is normally understood in terms of novel success, i.e., in terms of a theory's confirmed predictions of novel phenomena. Novel success has played a central role not only in discussions about theory choice (Lakatos 1978; Worrall 1989a; Scerri and Worrall 2001), but also in the realism debate: realists are normally willing to commit to the truth of only those theories which have managed to produce novel success (Worrall 1989b; Psillos 1999; Saatsi forthcoming). ${ }^{2}$

There is another form of fertility, which has received very little (if any) attention in the recent realism debate. This concerns theoretical fertility as construed by Ernan McMullin in several works. I shall call this form of fertility M-fertility. Although M-fertility is a much less restrictive notion than novel success - rendering it compatible with more potentially undesirable cases-McMullin has argued that it provides "perhaps the strongest grounds

\footnotetext{
${ }^{1}$ The other four virtues Kuhn identified are empirical accuracy, simplicity, unifying power or scope, external and internal consistency.

2 This has also to do with realists' reaction to the threat posed by the Pessimistic Meta Induction by Laudan (1981). For a recent discussion see Vickers (2013).
} 
for the thesis of scientific realism" (McMullin 1985, 264). Given how much controversy has come to surround the novel success-truth connection lately (Magnus and Callender 2004; Saatsi and Vickers 2011), and given that retrospective identification of success-generating theory-parts is potentially colored by our current theories (Stanford 2006), the realist should be happy for any principled argument that might diversify her arsenal.

This paper reviews and assesses M-fertility and its realist rationale in detail. I argue first that Daniel Nolan's objections against M-fertility as a theoretical virtue "in its own right" fail and, second, that McMullin's preferred example of the Bohr-Sommerfeld model of the atom does not support realism. At the same time, I shall suggest, the development BohrSommerfeld model can teach us important methodological lessons about theory appraisal.

The paper will proceed as follows. After briefly reviewing the standard notions of novel success in Section 2, I introduce M-fertility in Section 3. In this section, I also review Nolan's objection to M-fertility actually being a virtue and reject the claim that M-fertility is a virtue only in so far as it reduces to novel success, amongst other things, by emphasizing an aspect of M-fertility that is entirely neglected by Nolan, namely the idea of de-idealisation (3.2). In Section 3.3 I point out that the concept of de-idealisation in fact offers a welcome clarification of Lakatos' notoriously vague idea of a research programme's positive heuristic. In Section 4 I argue in detail that the development of the Bohr-Sommerfeld model of the atom-McMullin's and Lakatos's preferred example-does not support McMullin's idea of de-idealisation. In Section 5 I contrast the fertility of the Bohr model with the lack thereof in the case of the kinetic theory of heat and the so-called specific heat anomaly. In Section 6 I conclude my discussion.

\section{Standard notions of fertility as novel success}

The thesis that successfully predicted evidence ought to count more in the confirmation of theories than successfully accommodated evidence is also known as predictivism. Two important forms of predictivism have been proposed. According to temporal predictivism evidence $\mathrm{E}$ is novel with regard to a theory $\mathrm{T}$ entailing $\mathrm{E}$, when $\mathrm{T}$ was devised before $\mathrm{E}$ was discovered. According to heuristic predictivism evidence $\mathrm{E}$ is novel with regard to a theory $\mathrm{T}$ entailing $\mathrm{E}$ when $\mathrm{E}$ was not used in the construction of $\mathrm{T}$.

Heuristic predictivism has been defended mostly by John Worrall in several publications (e.g. 1989a; 2002). Worrall has argued that there is no rationale for temporal predictivism: there is no reason for why the bare time order between theory and evidence ought to somehow bear meaningfully on how well the theory is confirmed by the evidence. Heuristic predictivism, Worrall claims, does offer a plausible rationale: one ought not to accommodate evidence in an ad hoc fashion. When one uses evidence in the construction of a theory entailing that evidence, in contrast, one can easily ensure that the evidence is entailed by that evidence. The evidence cannot possibly pose a challenge to the theory and accordingly that evidence should not be allowed to boost our trust in the theory. 
Yet, temporal and heuristic predictivism need not be understood as contrasts. On the contrary, heuristic novelty is perhaps best understood as a logically weaker form of temporal novelty. That is, evidence that is temporally novel is automatically use-novel, but not vice versa. That is so, because evidence $E$ that was not known at the time when the theory T entailing E was constructed could of course not have be used in the construction of $\mathrm{T}$. Conversely, evidence known at the time when $\mathrm{T}$ was constructed is by definition not temporally novel, but can very well be use-novel (cf. Schindler 2013).

Heuristic predictivism is however not just more permissive than temporal predictivism. Its scope seems different too. Whereas on temporal predictivism one may allow for nonnovel evidence to support a theory, whilst attributing a greater degree of confirmation to theories confirmed by temporally novel evidence, on heuristic predictivism novelty seems to be a necessary condition for confirmation: only evidence that is not accommodated in an ad hoc fashion can confirm a theory. Once again though, one may, within heuristic predictivism, privilege temporal novelty (because the evidence in question could not possibly be used for constructing the theory entailing the evidence) and thereby allow for some kind of asymmetry in confirmation (cf. ibid). ${ }^{3}$

\section{McMullinian fertility and Nolan's challenge}

In a string of publications, Ernan McMullin has argued for a form of fertility that is different from novel success (McMullin 1968, 1976, 1982, 1984, 1985). In one place McMullin characterizes a theory's fertility as the ability to "cope with the unexpected" (McMullin 1976, $423)$, in particular, "as new evidence becomes available" $(1968,391)$. We shall refer to this kind of theoretical fertility as M-fertility. In another place, McMullin specifies:

... the theory proves to have the imaginative resources [...] to enable anomalies to be overcome and new and powerful extensions to be made. Here it is the long-term proven ability of the theory or research program to generate fruitful additions and modifications that has to be taken into account. (McMullin 1982, 16)

To put it schematically, at first pass, a theory $\mathrm{T}$ is $\mathrm{M}$-fertile if $\mathrm{T}$ has resources for accommodating evidence $\mathrm{E}$, inconsistent with $\mathrm{T}$, by suggesting modifications of $\mathrm{T}$ so that a modified version of $\mathrm{T}, \mathrm{T}^{*}$, can accommodate $\mathrm{E}$. This definition will be made more precise in what follows.

M-fertility is incompatible with both of the standard notions of novel success. For temporally novel success, evidence E must minimally be unknown at the time at which theory $\mathrm{T}$ is devised. E must also be anticipated by $\mathrm{T}$. But that is clearly not the case for what McMullin has in mind. In M-fertility, modified T* accommodates evidence that could not be

\footnotetext{
${ }^{3}$ There are of course other novel success criteria than the ones I mention above. However, temporal and use-novel success are the standard criteria used in the realism debate (e.g. Psillos 1999). Although Leplin's A Novel Defense of Realism gets often cited, the novel success criterion developed therein has received severe and adequate criticism by Ladyman (1999). For a more detailed discussion of accounts of novel success see my (Schindler 2013).
} 
accommodated by the original $\mathrm{T}$ (an anomaly for $\mathrm{T}$ is by definition not anticipated by $\mathrm{T}$ ). In M-fertility also use-novelty is violated: $\mathrm{T}$ is modified in order to accommodate E. Hence, in so far as M-fertility is a virtue, it is a virtue that is independent of the virtue of novel success. But is M-fertility really a virtue?

Before we proceed, let us also note that McMullin distinguishes between $\mathrm{P}$ (roven) and $\mathrm{U}$ (nproven) fertility. It is the former with which McMullin is mainly concerned. U-fertility, McMullin describes as a theory's "heuristic potential", or the "as-yet unexplored heuristic possibilities of the theory“ being high (McMullin 1976, 424). That is, a theory that is U-fertile has not yet been shown to be capable of accommodating anomalies, but might nevertheless hold to potential to do so. For McMullin, U-fertility is a non-epistemic virtue and therefore of little interest to him. ${ }^{4}$ We shall follow McMullin and lay our focus on P-fertility.

Accordingly we will read M-fertility as P-fertility by default.

\subsection{Nolan's challenge}

Nolan (1999) has questioned that there is a feasible rationale for viewing M-fertility as a virtue "in its own right". Nolan writes:

On the face of it, it can be a little hard to see why the liability of a theory to require improvements, or to raise new problems, should be considered a good thing. It is as if 'Faces many problems' or 'Could do better', or 'Much room for improvements' are high praise on the report card of a theory. Surely being in difficulties is not what we would expect of an ideal theory? (267)

"Kuhnian reasons" for thinking that M-fertility is a virtue, such as "opportunity to do science", intellectual challenge (267), and "employment prospects" (268), Nolan rejects as unconvincing - rightly so. ${ }^{5}$ Although these reasons might be good pragmatic reasons for valuing a theory, they are not epistemic ones. And those we ought to be after, in the philosophy of science.

Nolan does not deny M-fertility any value. However, he denies M-fertility a value "over and above the virtues which in fact its value rests [upon]" (265). In other words, Nolan is a reductionist about M-fertility.

Although Nolan, like McMullin, makes P-fertility the main focus of his discussion, his reductionism extends also to U-fertility. For Nolan U-fertility is "the potential to advance, or improve, or progress" and is valuable "as a means, rather than an end" where the end is some other virtue (271; original emphasis). Nolan is fairly unspecific about the virtues Ufertility might be a means for. However, he does mention in passing "increasing strength, increasing predictive power and accuracy, increasing unification" (270). U-fertility is then

\footnotetext{
${ }^{4}$ McMullin uses the idea of U-fertility mainly to describe and criticize Lakatos's account. See Section 3.3 for a comparison of the two accounts.

${ }^{5}$ Denoting such sociological aspects of science as "Kuhnian" seems slightly unfair. Contrary to common misinterpretations, Kuhn thought there was much more (cognitive) involved in the pursuit of normal science than just sociological opportunity. There is however some textual evidence that Kuhn, at least in his later work, thought of fertility also in sociological terms (Kuhn 1977, 322 fn 6).
} 
the "chance that a close successor or a later theory-stage will be significantly better than the current one" with regards to these (or other) virtues (271).

With regards to $\mathrm{P}$ - or M-fertility (recall that we treat those as equivalents), Nolan is much more specific: he attempts a reduction of M-fertility to novel success. Given that, as mentioned above, M-fertility and novel success are incompatible, this should come as a surprise. Nolan's reduction therefore requires a little tweak.

Nolan invites us to consider a sequence of related theories $V_{1}, V_{2}, V_{3}$, etc. Each of those gets revised after the fact, we suppose, on the basis of some anomalies so that they each eventually accommodate those anomalies (ibid.., 273). Crucially, Nolan proposes that we associate with these specific theory developments a meta-hypothesis $\mathrm{V}$, for example, the meta-hypothesis that "some atomic theory of gases is true", or that "the best available theory is going to lie in the tradition of $\mathrm{V}^{\prime \prime}$. Now,

$[\ldots]$ the [meta-]hypothesis that the V-series of theories is on the right track (or some V-like theory is true, or close to the truth, or the best theory of the phenomena available) may be doing very well nonetheless, and may even be confirmed by the evidence which refutes specific versions. (ibid. 276; added emphasis)

In other words, evidence that looks anomalous from the perspective of the specific Vtheories can be viewed as successes from the perspective of the meta-hypothesis $\mathrm{V}$. What is more, since the meta-hypothesis came to life with the first specific $\mathrm{V}$ theory (at the latest), we can view all those anomalies for the specific $V$-theories as novel evidence for the meta hypothesis V: "[...] the fact that V-like theories got better and better counts as novel confirmation for the theory that V-like theories are good" and "on the right track" (276). ${ }^{6} \mathrm{Or}$ so Nolan suggests.

Several things about Nolan's proposal should strike one as dubious. First of all, the sort of novel success Nolan claims for meta-hypotheses is a deflationary one. Normally, we have reason to be impressed with a theory's novel success because the predicted phenomena are precisely as predicted by the theory (see Section 2). But Nolan's meta-hypotheses give us no such reasons. On the contrary, Nolan's meta-hypotheses are extremely vague: they are compatible with all sorts of evidence. Relatedly, although it is true that "[...] the fact that this general claim [i.e., the meta-hypothesis] was advanced before the history of specific versions renders it able to have novel confirmation" (278), this is not sufficient for novel success. That is, it is not sufficient for $\mathrm{E}$ to count as novel success for $\mathrm{T}$ that $\mathrm{E}$ comes chronologically before T; E must be predicted by T. But that is clearly not the case. The meta-hypothesis does not predict any of the anomalies faced by the specific versions. If it did, then the anomalies would not count as anomalies in the first place! So even if one were to be persuaded that the anomalies of the specific $\mathrm{V}$-theories might be evidence for the meta-hypothesis $\mathrm{V}$, one will be at a loss as to why these pieces of evidence ought to count as novel success for the metahypothesis. Second, Nolan's appeal to meta-hypotheses is highly arbitrary. Rather than

\footnotetext{
${ }^{6}$ Nolan suggests that the meta-hypotheses may be construed as some kind of "general theory" instead (278f.). This difference is minor and shall not concern us here.
} 
choosing "some atomic theory of gases is true" Nolan offers us no constraints for why we cannot choose meta-hypotheses at even higher levels of abstraction, as for example "all matter is made up of atoms" or "unobservables exist"? In comparison to those hypotheses, any accommodative success in the realm of chemistry and physics concerning atoms and molecules would count as temporally novel success on Nolan's account. But that is clearly absurd. Thirdly, Nolan pretty much ignores the fact that McMullin does offer a rationale for why M-fertility ought to be a virtue. ${ }^{7}$ Let us have a closer look.

\subsection{Non-ad hoc modifications through de-idealisations}

For McMullin, not any old modification of a theory may count in its favor, when gauging its empirical success. On the contrary, and minimally, modifications of a theory in response to an anomaly are only allowed when they are not ad hoc (e.g.McMullin 1985, 264). And modifications of a theory in response to anomalies are not ad hoc, in turn, when the modifications result from de-idealizations of the original theory. In a good theory, McMullin surmises, these de-idealizations are suggested by the original theory itself:

If the model is a good one, these processes [of self-correction] are not ad hoc; they are suggested by the model itself. Where the processes are of an ad hoc sort, the implication is that the model is not a good one [...] (McMullin 1985, 264)

This is to be understood thus: although scientists are very well aware of the fact that the assumptions underlying an initial model / theory might be unrealistic, they leave out complications in the first steps for the sake of simplicity (cf. McMullin 1968, 394). In sum then, M-fertility may thus be defined as a theory's ability to accommodate anomalies in a non-ad hoc fashion by means of de-idealisation of its original simplifying assumptions. ${ }^{8}$ Put schematically, a theory $\mathrm{T}$ is $\mathrm{M}$-fertile if an anomaly $\mathrm{E}$ to $\mathrm{T}$ can be accommodated by deidealising $\mathrm{T}$ to $\mathrm{T}^{*}$ so that $\mathrm{T}^{*}$ entails (or makes likely) $\mathrm{E}$. Why is this a virtue? There are two, fairly independent, reasons to be found in McMullin's work.

First, M-fertility is a virtue because it allows accommodating apparent anomalies in a non-ad hoc fashion, and non-ad hocness clearly is a methodological desideratum. There is a separate question of whether non-ad hocness increases the chances of a theory being true. This, I'm not so sure about. What is uncontroversial, however, is that a theory that is ad hoc with regard to some evidence should receive less confirmation from that evidence than a theory that accommodates this evidence in a non-ad hoc way. Confirmation is also an

\footnotetext{
${ }^{7}$ Another criticism of Nolan's proposal, to be found in Segall (2008), is that "any detailed examination of the history of a long-standing successful theory is likely to show that it is the specifics of the current theoretical and experimental situation that leads to the next development" (244).

${ }^{8}$ McMullin, on several occasions, attributes a theory's fertility to the model associated with a theory. McMullin has a peculiar view of models. For him, "[t]he theory is derived from the model [...] not the reverse [...] the theory is about [a particular] model and about nothing else" (McMullin 1968, 389). At least the latter part of this view seems to come close to Cartwright (1983)'s 'prepared descriptions' of real systems, which a theory is about, rather than about the real system itself. At the same time, McMullin (ibid.) is quite adamant in his rejection of some of Cartwright's (antirealist) conclusions. The differences between models and theories shall not concern us here.
} 
epistemic matter. So non-ad hocness would seem to be epistemic at least in a minimal, nonrealist, sense.

Second, M-fertility is a virtue on McMullin's account, because it indicates to us that the original model we started out with got something fundamentally right about nature:

This technique [of de-idealisation] will work only if the original model idealizes the real structure of the object. (McMullin 1985, 261) Without such a [rough] fit [between the original model and the real structure of the object], there would be no reason for the model to exhibit this sort of fertility. This gives perhaps the strongest grounds for the thesis of scientific realism. (ibid., 264)

In other words, the approximate truth of the original model explains why the changes suggested by the model itself help it to overcome anomalies.. Although this resembles the standard explanationist defense of realism, McMullin's explanandum is different (M-fertility instead of novel success).

Thus, there is not one but actually two rationales McMullin offers for M-fertility. It's quite surprising that Nolan, in his discussion of M-fertility, makes no mention of either of these.

\subsection{M-fertility and Lakatos' positive heuristic}

Before we proceed with the assessment of the the rationales of M-fertility offered by McMullin, it is worth dwelling on their strengths: not only do they make it plausible that Mfertility is a virtue in its own right, but, as we shall see in the current section, they also seem to offer clarification with regards to related accounts, such as Imre Laktos's ides of research programmes. The comparison will also give us a better sense of the role of anomalies in McMullin's account.

For Lakatos, research programmes (basically a theory-development over time) consist of a 'hard core' of 'irrefutable' assumptions and a 'protective belt' of auxiliary assumptions, which "bear the brunt of tests and get adjusted and re-adjusted, or even completely replaced, to defend the thus-hardened core" (133). The so-called 'negative heuristic' of research programmes "forbids us to direct the modus tollens at this 'hard core'", in contrast to the 'positive heuristic', which guides scientists in the construction and modification of the protective belt. Lakatos describes the positive heuristic also as providing 'instructions' for building "ever more complicated models simulating reality" (133-5). Although this is very vague, Lakatos' examples suggest that he has something very similar in mind as McMullin's idea of de-idealisation. Newton, for example, first assigned infinite mass to the sun as the central body in the solar system in order to calculate planetary orbits on the basis of the law of gravitation. Later, he relaxed this assumption so that the sun and the planets would receive a common centre of gravity. Furthermore Newton first treated the planets and the sun as mass-points and only later as mass-balls. All these changes were reasonable and expected changes. Just like they are in the de-idealisations McMullin talks about. Indeed McMullin seems to have the same thing in mind as Lakatos. A difference between McMullin's and Lakatos's account, however, is the emphasis that Lakatos places on 
the disregard of anomalies and on the novel success in the evaluation of research programmes.

Whereas in M-fertility anomalies 'trigger' de-idealisations, they have no place in the development of a research programme in Lakatos's view. Ideally, for him, "[...] the positive heuristic forges ahead with almost complete disregard of 'refutations' [...]" (137). Indeed, Lakatos goes as far as saying that "relatively few experiments are really important" for the development of a research programme, as long as it is progressive (151). In contrast, when scientists do start caring about modifying the research programme so as to accommodate anomalies, the research programme is degenerating:

Which problems scientists working in powerful research programmes rationally choose, is determined by the positive heuristic of the programme rather than by psychologically worrying (or technologically urgent) anomalies. The anomalies are listed but shoved aside in the hope that they will turn, in due course, into corroborations of the programme. Only those scientists have to rivet their attention on anomalies who are either engaged in trial-and- error exercises or who work in a degenerating phase of a research programme when the positive heuristic ran out of steam. (Lakatos 1970, 137, added emphasis)

Lakatos also requires consistent theoretical progress for a progressive research programme, that is, he requires that any modification of a research programme results in novel predictions (134). And although Lakatos demands only intermittent empirical progress, i.e., confirmations of the novel predictions of a research programme, ultimately research programmes must produce novel success for them to be considered progressive (ibid.). Once a research programme does manage to produce novel success, this will have positive, retrospective, bearing on the assessment of the entire research programme, regardless of how many anomalies the programme encountered hitherto: "a long series of 'refutations'" can therefore be turned "into a resounding success story" (ibid.).

Lakatos is quite explicit that the notion of novelty he has in mind is temporal novelty. That is, the same piece of evidence can count towards the progressiveness or degeneracy of a research programme, depending on whether that piece of evidence was predicted (in the temporal sense) by the research programme or whether it was already in place when the research programme was developed (151-2). Lakatos's demand that theoretical progress be consistent is in fact motivated by the value he places on novel success: only a research programme that 'forges ahead', before the evidence relevant to its predictions are gathered, can give itself a chance of having temporally novel success.

Thus, although there are clear similarities between M-fertility and Lakatos's progressive research programmes (in particular, theoretical change through de-idealisation) a crucial dissimilarity is Lakatos's insistence on (ultimate) novel success as a necessary condition for theoretical progress. On McMullin's account, by contrast, since changes to the theory follow the encounter of anomalies, both temporally novel success and use-novel success criteria are violated.

Of course, if de-idealisation is what justifies the changes to a theory both in McMullin's and Lakatos' account, then it seems merely a contingent matter that, in some 
cases, changes to the theory are made after the encounter of anomalies and, in other cases, changes are made before anomalies are faced. At bottom there is simply some kind of intrinsic necessity to de-idealise, regardless of whether the evidence that is being accommodated comes in before or after the de-idealisation is actually made. Lakatos' insistence on temporal novel success seems misplaced. Indeed, if the rationale for M-fertility is sustainable, then the realists' obsession with novel success seems likewise gratuitous.

\section{The Bohr-Sommerfeld model of the atom}

Despite the apparent plausibility of the idea of de-idealisation, it doesn't seem to capture what is really going on in an important historical case, indeed in a historical case that has been used both my McMullin and Lakatos as the paradigmatic example of their accounts. This case concerns the Bohr-Sommerfeld model of the atom, which envisages atoms as small planetary systems with electrons orbiting the atomic nucleus, and which was expounded by Niels Bohr in his famous triology in the Philosophical Magazine in 1913 and substantially developed by Arnold Sommerfeld in the period of 1913-1925 (McMullin 1968, 393-5; Lakatos 1970, 146ff.; 1985, 259ff.).

Conveniently both McMullin and Lakatos divide the historical development of the BohrSommerfeld model into similar de-idealisation stages, each of which allowed the model to accommodate certain anomalies, and each of which, according to Lakatos (ibid.), "was planned right at the start" and which according to McMullin had to happen (1968., 394-5): ${ }^{9}$

1. Assigning finite mass to an initially infinite mass atomic nucleus. This change helped to account for the Pickering-Fowler spectral lines of ionized helium.

2. Turning circular electron orbits into elliptic orbits. This allowed the model to account for the so-called Stark effect, i.e., the splitting of spectral lines in an electric field.

3. Adding (realistic) relativistic effects to the elliptic electron orbits. This accounted for the fine structure of hydrogen.

A final stage in the development of the Bohr-Sommerfeld model is mentioned by both McMullin and Lakatos, but treated slightly different from the previous three stages (details to be discussed below).

4. Introducing the concept of electron spin, as suggested by Goudsmit and Uhlenbeck in 1925, in response to the anomalous Zeeman effect.

As we shall see in the following, none of these stages supports the idea of accommodation of anomalies / novel predictions through de-idealisation. Thus, contra McMullin, none of these stages can support realism (by McMullin's own lights).

\footnotetext{
${ }_{9}^{9}$ The similarities of McMullin's and Lakatos's account are indeed striking. Who was first and who might have been inspired by whom is not subject of this paper though. For an attempt to work out differences beyond the ones emphasized in this paper see McMullin (1976). With regards to the Bohr model, McMullin and Lakatos's accounts are not equally detailed in all respect. I shall use something like the best reconstruction.
} 


\subsection{Stage 1: finite mass nucleus and the Pickering-Fowler series}

\section{The facts}

In 1897 the American astronomer E. Pickering measured several spectral lines emitted from the star $\xi$-Puppis. Because those lines converged to the same limit as the Balmer series, which described the spectral lines of hydrogen, they too were ascribed to hydrogen. In 1912 the British astrophysicist A. Fowler managed to reproduce the $4686 \AA$ line, which Pickering had obtained (amongst other lines) from $\xi$-Puppis earlier, in the laboratory with a discharge tube containing a mixture of hydrogen and helium. Contrary to the Bohr model, these additional lines seemed to suggest half-integral quantum numbers, whereas the Bohr model only allowed for integral ones. Bohr, however, speculated that Pickering's and Fowler's measurements were not caused by hydrogen but rather by ionized helium. He therefore calculated the expected lines with an atomic model containing two protons and one electron, and was able to derive Pickering and Fowler's results. Indeed, Bohr predicted that the relevant spectral lines would be measured even when chlorine instead of hydrogen were to be used as a catalysator. This prediction was confirmed by Evans on September 4, 1913. The remaining small discrepancy between Bohr's predictions for ionized helium and Fowler's data was largely removed by assigning finite mass to the atomic nucleus, allowing for a small motion of the nucleus around a common centre of mass due to the (minimal) attraction of the nucleus by the electron. ${ }^{10}$

\section{Assessment}

Lakatos calls Bohr's increase of the atomic number of the model a "monster-adjustment", which he describes as "turning a counterexample, in the light of some new theory, into an example" (ibid., fn 1, p. 149). Lakatos tries to justify this "monster-adjustment" retrospectively by the ensuing novel success. More specifically, Lakatos singles out Bohr's prediction that the Fowler line should be observable in tubes containing no hydrogen at all, but only helium and another catalysator (ibid., fn. 1, 149). And indeed, it was reported that Einstein called this confirmation "an enormous achievement" and concluded that "the theory of Bohr must then be right" (von Hevesy in a letter to Bohr on 23 September 1913Hoyer 1981, 531).

First of all, one may question whether increasing the atomic number of the model really constitutes a change of the model in the first place (let alone a "monsteradjustment"). After all, the basic assumptions seem to be fairly unscathed; it is simply applied to a different chemical element, namely helium. Second, even if we were to accept this as a change of the model, it is hard to see how applying the model to a different element could count as a de-idealisation. And third, it is at least questionable whether the

\footnotetext{
${ }^{10}$ In fact, following Fowler's reaction to Bohr's suggestion, Bohr proposed a further correction to his model, namely the velocity dependence of the mass of the electron (Bohr's letter to Fowler on April 15, 1914, in: Hoyer 1981, 504ff.). This, to my knowledge, is the first occasion at which Bohr considered relativity corrections in writing. See also the next stage discussed in the main text of this paper.
} 
successful prediction of the Fowler line in tubes containing no hydrogen at all can count as genuine novel success. Certainly in the realism debate, novel success is understood more restrictively as the prediction of phenomena that our currently accepted background theories tell us nothing about. However, both hydrogen and helium were of course known chemical elements at the time. In spite all of this, Bohr's theory was received extremely positively in some quarters, even before the discovery of the Fowler line. For example, Sommerfeld wrote to Bohr on September 4 in 1913:

Although I am for the present still a bit skeptical about atomic models, your calculation of the constant [in the long-known Balmer formula] is nevertheless a great achievement. (ibid., 603)

Overall, the idea of de-idealisation appears not to underlie physicists' positive reception of the Bohr model with regards to the anomaly of the Fowler line.

\subsection{Stage 2 and 3: ellipses, the Stark effect, and the fine structure}

Both McMullin and Lakatos present the move from circular to elliptical orbits as a matter of course, that is, as an almost necessary change in de-simplification of the model that was expected to happen even regardless of any anomalies. McMullin for instance writes that "there is no reason to restrict the electron to circular orbits, since elliptical orbits are the normal paths for bodies under central forces of this type" $(1968,394)$ and

If one has a two-body system of the sort postulated, one will have to have nuclear motion, elliptical orbits, and relativistic mass effects, unless the laws of physics, known from elsewhere are assumed not to apply to the entities in the model (ibid., 394-5; original emphasis). ${ }^{11}$

The funny thing is, of course, that the Bohr-Sommerfeld model was a model of entities which did behave very differently from what the laws of physics appeared to dictate elsewhere; stationary electron orbits were irreconcilable with the classical laws of physics. It is thus odd to appeal to the regular laws of physics to motivate changes to the model. One might even question whether the move from circular to elliptical orbits is accurately described as a deidealisation. After all, circular orbits are just a special case of elliptical orbits. ${ }^{12}$ At any rate, we shall see in a moment that elliptic orbits by no means replaced circular orbits; they complemented them. What is more, circular orbits were explicitly deemed more likely than elliptic orbits. None of this makes sense on a de-idealisation story.

What is also striking is that one finds no comments by Sommerfeld, Bohr and others, which would give indication that their motivation for introducing changes to the model had anything to do with de-idealisation. Instead, as we shall see in the next section, the only motivation for introducing ellipses stated by Bohr, who toyed with the idea in 1915, and by Sommerfeld, who delivered a detailed treatment in 1915-1916, was the accommodation of

\footnotetext{
${ }^{11}$ Lakatos writes that the change was "as planned" (ibid. 148).

${ }^{12}$ One might perhaps retort that circular motion is more unlikely than elliptical orbits insofar as in circular motion centripetal forces must precisely counterbalance centripetal acceleration. Circular motion would then be an idealization of elliptic motion in that, normally, conditions are such that centripetal forces and centripetal acceleration would not counterbalance each other in nature.
} 
the fine structure of hydrogen and other elements. In other words, all that Bohr and Sommerfeld seemed to care about when designing their models was empirical adequacy.

\section{The facts}

Already in 1887 it was discovered by Michelson and Morley that one line in the spectrum of hydrogen was not a singlet, as described by the Balmer series, but rather a doublet. The importance of this finding remained unappreciated and appears to have received renewed attention only with the arrival of Bohr's model. In 1914 W.E. Curtis refined Michelson and Morley's observations and concluded that "Balmer's formula has been found to be inexact" (Curtis 1914, 620) and that Bohr's derived spectral lines formula, corrected for the finite nucleus mass and the velocity dependence of the mass of the electron (cf. fn. 10) could not accommodate the results either (616). The suggestion was made that these results could be accounted for by the magnetic properties of the atomic nucleus. Bohr, in a discussion note, dismissed this proposal. Instead he pointed to a mistake by Curtis and re-iterated the idea of relativity corrections for circular electron orbits. He admitted though that this would account only for $1 / 3$ of the deviations from the Balmer series, as observed by Curtis. Bohr conceded to Curtis that his earlier idea of explaining the doublet of hydrogen as "not true doublets" and as resulting from the presence of an electric field was incorrect. He continued that "there is perhaps another way of explaining the observed doubling without introducing new assumptions as to a complicated internal structure of the hydrogen nucleus". What he was pondering was the supposition "that we would obtain a doubling of the lines [of hydrogen] if the orbits are not circular". In view of "great number of new assumptions involved in such a calculation" he preferred to "await more accurate measurements" (Bohr 1915).

Sommerfeld took a more proactive approach. In the same year in which Bohr wrote his discussion note, Sommerfeld presented a detailed treatment of elliptic orbits for the Bohr model to the Bavarian Academy of Sciences, which was then published in the following year in the society's protocols (1916b) and, in more extended form in (1916a). In the latter, Sommerfeld set out to "extend significantly" Bohr's theory with a "consideration of noncircular orbits" in order to "shed light on the 'Sonderstellung' [i.e., peculiar status] of spectral lines of hydrogen", which consisted in the fact that, whereas other elements had different line series (principal, subordinate, and combinatorial) and different line types (singlets, doublets, and triplets), hydrogen had just a single Balmers's series. His basic idea was this:

According to the view to be presented here this is to be explained by the fact that [in the hydrogen spectrum] a number of [different] series coincide in the Balmer series [of hydrogen], i.e. each of its [i.e. hydrogen's] lines can be produced in a number of different ways, not only through circular motion but also through elliptic orbits with particular eccentricities, as can be shown experimentally. (ibid., 4; my emphasis)

Interestingly Curtis, although not mentioned by Sommerfeld, had developed a very similar idea, not on theoretical, but on comparative grounds. The relevant passage is worth quoting in full for clarification: 
As [the Sharp series of hydrogen] have never been seen, however, it is reasonable to suppose that in the case of hydrogen the Sharp and Diffuse series practically coincide, the Balmer series representing the superposition of the two. There is not at present any direct evidence bearing on this question, but the above manner of regarding the Balmer series has the advantage of bringing the hydrogen spectrum into line with other series spectra, and converting it from an exception into a limiting case. (Curtis 1914, 619, added emphasis)

Thus, both Curtis and Sommerfeld sought to systematize their observations regarding the spectral lines of the elements in such a way that hydrogen would not stand out as a singularity and so that it would rather "be brought into line" with the other elements and its peculiarities explained with the same theoretical resources used for the other elements. ${ }^{13}$

In his theoretical treatment, in addition to Bohr's quantum number $n$, quantizing the size of circular orbits, Sommerfeld introduced another quantum number (referred to him as $n$ ') for the quantisation of 'shape' of the orbits, or more precisely, its eccentricity. By way of phase integrals Sommerfeld was then able to relate both quantum numbers to the eccentricity of elliptic orbits, as described by the following formula

$$
1-\varepsilon^{2}=\frac{n^{2}}{\left(n+n^{\prime}\right)^{2}}
$$

This allowed him to derive an expression for the energy of the system so that "the energy is thus uniquely determined by the sum of the quanta of action $\left[n+n^{\prime}\right]$, which we may distribute arbitrarily among the azimuthal and radial coordinates" (Sommerfeld 1916a, 19). Sommerfeld called this result of "penetrating certainty" (schlagende Bestimmtheit). As can be seen from the above equation, if the newly introduced quantum number $\mathrm{n}^{\prime}$ would be zero, the eccentricity would turn zero too, rendering the electron orbit circular. And this possibility Sommerfeld did allow. Thus, rather than circular orbits replacing elliptic orbits in the Sommerfeld model, the former complemented the latter. What is more, in his treatment of different line intensities, Sommerfeld even went as far as rendering circular motion more likely:

We have assumed that the circular orbit is the most likely and that the elliptic orbit is the more likely the bigger its eccentricity. (Sommerfeld 1916a, 63)

With the above formula, Sommerfeld was now able to accommodate what he conceived of as the "Sonderstellung" of hydrogen with the theoretical machinery that also accommodated the spectral lines of other elements. The amended Balmer series formula, containing two quantum numbers $v=N\left(\frac{1}{\left(n+n^{\prime}\right)^{2}}-\frac{1}{\left(m+m^{\prime}\right)^{2}}\right)$, for hydrogen would "reduce, in a sense, by accident" to the standard formula containing only one quantum number, as derived originally by Bohr (20). ${ }^{14}$ Sommerfeld concluded that the Balmer series could now "appear in a new light", as it could now be viewed as being caused in a number of different ways

\footnotetext{
${ }^{13}$ There were further precursors. The attempt to integrate the hydrogen lines into the lines of other elements has been tracked historically by Robotti (1983). Curiously, neither Curtis nor Sommerfeld play any part in Robotti's story.

${ }^{14}$ In this formula, $n, n^{\prime}$ describe the initial electron orbit and $m, m^{\prime}$ the final electron orbits (i.e., before and after a quantum 'jump' of the electron).
} 
(with the second quantum number $\mathrm{n}^{\prime}$ taking different values without this translating into different spectral lines). This, for Sommerfeld, had "deepened theoretical significance" in comparison to Bohr's theory (Sommerfeld 1923, 237). ${ }^{15}$ At the same time, Sommerfeld pointed out that the "different ways of production" ("Erzeugungsarten") coincided in a single Balmer line "only approximately" and could be retrieved by means of including relativistic effects (Sommerfeld 1916a, 25). Sommerfeld then dedicated the entire second part of his treatment to the fine structure of hydrogen, and in particular to the hydrogen doublet. Crucially, Sommerfeld was able to derive the correct fine structure constant for hydrogen, which has been described as "perhaps the most remarkable numerical coincidence in the history of physics" (R. Kronig cited in Pais 1991, 188). The historian Kragh has concluded that "by some sort of historical magic, Sommerfeld managed in 1916 to get the correct formula from what turned out to be an utterly inadequate model" (Kragh 1985, 84). ${ }^{16}$

\section{Assessment}

We can make two crucial observations regarding this stage of the development of the BohrSommerfeld model. First, the changes made to the model are inconsistent with both novelty criteria. Second, the introduction of elliptic motion into the model can hardly be understood in terms of de-idealisation. Let us first attend to the second point.

For McMullin, the introduction of circular orbits amounts to almost a physical necessity in the goal of de-idealising the model. And yet we observed that circular motion still figures in Sommerfeld's development of Bohr's model. What is more, Sommerfeld deems it even more likely in order to make sense of line intensities.

Regarding the first point, we note that the motivations stated by Bohr and Sommerfeld for the change from circular to elliptic orbits by the scientists in question were clearly governed by the attempt to accommodate the fine structure of hydrogen and other elements. On the standard accounts of novel success, this change must therefore be considered ad hoc: the model was changed in order to accommodate the fine structure, i.e., the evidence was used in the construction of the model that accommodated that model. That is a violation not only of use-novelty but also of temporal novelty, for, recall, the latter is logically stronger than the former.

Despite the violation of the novelty criteria and the idea of de-idealisation being at odds with the actual development by Sommerfeld, Sommerfeld's model got an overwhelmingly positive reception. Einstein, for example, in a letter to Sommerfeld in February 1916, called Sommerfeld's treatment 'a revelation', which 'delighted' him and which convinced him that Sommerfeld was 'right' (Einstein in Eckert and Märker 2000, 5245). About half a year later, Einstein wrote to Sommerfeld in August 1916:

\footnotetext{
15 The Stark effect was accounted for by the introduction of a third quantum number, quantising the "location of the orbit" relative to an external electric field. This was done successfully by Sommerfeld's student Epstein and by Schwarzschild. See e.g. Kragh $(2012,154)$ for details. ${ }^{16}$ For a philosophical discussion of this achievement see Vickers (2012).
} 
Your investigation of spectra belongs among my most beautiful experiences in physics. Only through it do Bohr's ideas become completely convincing. (ibid., 563)

Bohr, likewise, said on 19 March 1916: "I do not believe ever to have read anything with more joy than your beautiful work" (ibid. 603). And H.A. Lorentz even commented to Sommerfeld on 14 February 1917: "You have reached one of the most beautiful results in theoretical physics" (ibid., 574).

Have we perhaps treated novelty defenders unfairly? Should we not rather ask whether the changes made to the model ultimately resulted in novel success, rather than whether the changes themselves can count as novel success? This, at least, is what Lakatos would recommend. On Lakatos's account prima facie methodologically suspect changes such as modifications in response to anomalies, recall, can be justified retrospectively by novel success generated by the research programme. And indeed Sommerfeld's model did occasion confirmations of novel predictions. In particular, Sommerfeld correctly predicted the fine structure of ionized helium, as confirmed by Paschen (1916). Yet neither Einstein's nor Bohr's assessment could have been influenced by this confirmation: both were made before Paschen's results. ${ }^{17}$ Furthermore, not only was the accuracy of Paschen's measurements challenged by new high precision measurements by the German physicists E.J. Gehrcke and E. Lau in early 1920, but also, as Kragh (1985) has pointed out, any empirical test of Sommerfeld's model was problematic, because it depended on rather arbitrary intensity rules, such the one mentioned above (ibid., 73 and 93). Thus, whether retrospective or not, novel success does not seem to be able to licence the changes to the Bohr-Sommerfeld model. For the standard, novel success-admiring, realist these changes must be impermissible ones.

\subsection{A material de-idealisation: electron spin}

The previous three stages are all examples for what McMullin considers 'formal' deidealisations, which he defines as de-idealisations of a model or theory which leaves out features in the theoretical representation of a target system that are known to be relevant to the explanation of the target system. The fourth stage of McMullin's discussion of the development of the Bohr-Sommerfeld model, the invocation of concept of spin by G. Uhlenbeck and S. Goudsmit in 1925, is an instance of what McMullin calls 'material' de-idealisation. In that kind of de-idealisation one adds features to the theoretical representation of the target system which were not known to be relevant in the explanation of the target system when the first model or theory was devised (McMullin 1985, 258). ${ }^{18}$ Now to the details.

\footnotetext{
${ }^{17}$ On 21 May 1916, Friedrich Paschen informed Sommerfeld that he could experimentally confirm the predictions of Sommerfeld's model about the fine structure to be expected in ionized helium. Paschen reported the results to Annalen der Physik in July 1916 where they, according to the publisher (personal communication), saw print probably in September of the same year.

${ }^{18}$ Both formal and construct idealisations are forms of construct idealisations, where simplification is "worked on" a "conceptual representation of the object", in contrast to causal idealisations, the "problem situation itself" is simplified, so that "the diversity of causes found in Nature is reduced and made manageable" (McMullin 1985, 255 and 265).
} 


\section{The facts ${ }^{19}$}

The introduction of a third quantum number had allowed Sommerfeld (with the help of his assistant Epstein) to account for not only the Stark effect, but also for the so-called 'normal' Zeeman effect, i.e., the splitting of lines in in the presence of a magnetic field. This effect that was known since 1897 and had already explained by Lorentz in terms of his electron model, a feat for which he, together with Zeeman, received the Nobel Prize. In the same year, however, it was discovered that there were forms of the Zeeman effect, specifically in sodium, and generally in elements producing multiplet spectral lines, which did not conform to Lorentz's predictions. For that reason it came to be known (to this day) as the 'anomalous Zeeman effect'. In fact, the anomalous Zeeman effect constituted a major problem also for the Bohr-Sommerfeld theory. In order to accommodate it, Sommerfeld introduced yet another quantum number in 1920. In contrast to the other three quantum numbers, Sommerfeld found no electron-orbit correlate for it. Instead he attributed it to a somewhat mysterious 'hidden rotation', which, due to the works of A. Landé and Heisenberg around 1921-22, was construed as an angular momentum of the inner 'core' or 'rump' of electrons, whereby the new quantum number quantized the states of (magnetic) interactions between that core and the single outer, valence, electron. With the help of this model and the introduction of half-integer quantum numbers, Landé, in 1921, managed to capture the line splitting data on the alkali metals, but only at the cost of counting the contribution of the rump to the energy of the atom, somewhat arbitrarily, twice. Pauli showed that Lande's solution, although empirically adequate, led to absurd consequences (cf. e.g. Jammer 1989, 137). Instead, Pauli proposed that the 'hidden rotation' is not to be found in the core, as assumed by Landé, Heisenberg, and others, but rather in the valence electron. The anomalous Zeeman effect, accordingly, would be then due to a classically nondescribable 'two-valuedness' (German: Zweideutigkeit) of the electron. Extrapolating this idea from the context of alkalis in 1924, Pauli assumed that his new-found Zweideutigkeit was a property not just of electrons in alkali atoms but of electrons in any atom. ${ }^{20}$

In an attempt to return to the classical visualisability of the Bohr-Sommerfeld model, in line with Sommerfeld's interpretation of quantum numbers as corresponding to degrees of freedom of the electron, Uhlenbeck and Goudsmit in 1925, and independently of them R. Kronig, interpreted Pauli's Zweideutigkeit as the literal spinning of electrons around their own axis on their orbits around the nucleus. ${ }^{21}$ The concept of electron spin explained not only the fine structure of hydrogen and the alkali elements more accurately than it was possible on the Bohr-Sommerfeld model, but it also accounted for the anomalous Zeeman

\footnotetext{
${ }^{19}$ This section is based on the extensive historical discussions that can be found in Pais (1991), Jammer (1989), Mehra and Rechenberg (1982), Kragh (2012), and Forman (1968).

${ }^{20} \mathrm{On}$ the basis of this, Pauli formulated the famous exclusion principle. See the abovementioned sources for details.

${ }^{21}$ The first paper which Uhlenbeck and Goudsmit published on electron spin appeared in Naturwissenschaften in 1925. A similar paper in English appeared in 1926 in Nature. See de Regt (2001) for a discussion of the role of visualisability in physics.
} 
effect. A discrepancy of a factor of 2 between the predictions derived from the concept of spin and the fine structure of hydrogen was removed by L. Thomas in 1926, who corrected a mistake in Uhlenbeck and Goudsmit's relativistic treatment of the electron. Another problem, associated with the latter, however, remained: the absurd consequence of the surface of spinning electrons being faster than the speed of light, resulting from assigning electrons an extension, needed for the classical spin property. This issue remained unresolved until Pauli's development of spin matrices within the framework of a nonrelativistic quantum mechanics in 1926. ${ }^{22}$

\section{Assessment}

The concept of electron spin, as put forward by Uhlenbeck and Goudsmit, was introduced in order to accommodate the Zeemann effect. Both novel success criteria are clearly violated.

Can the introduction of spin be understood as some kind of de-idealisation?

It is interesting to note, first of all, that McMullin and Lakatos disagree about whether or not the concept of spin was suggested already by the original model by Bohr. Whereas Lakatos believes that spin was "planned right at the start" alongside a finite mass nucleus and elliptic orbits (1970, 146), McMullin has it that, in the original model, "there was no reason to suppose that the electron would possess spin" (McMullin 1985, 263). ${ }^{23}$ It is questionable whether one can arbitrate in a meaningful way here save for pointing out that it would be strange if spin had been suggested by the original model and then not been considered for more than 10 years before Uhlenbeck and Goudsmit introduced the idea in 1925.

Despite his reluctance to consider spin as being suggested by the original model, McMullin does believe that the addition of spin ought to count in the favour of the BohrSommerfeld's fertility. This is slightly strange given that McMullin in his account stipulates de-idealisation as a necessary condition for changes not to be ad hoc. On the contrary, he concludes in favor of the fertility of the Bohr-Sommerfeld model by saying that "from this simple physical assumption the correct answers were found for both Zeeman effects, not only in hydrogen but in other types of atom as well" (McMullin 1968, 395).

In stark contrast to McMullin's positive assessment of the introduction of spin being a fertile modification, Lakatos concludes his brief remarks about the introduction of spin by saying that "the temerity in proposing wild inconsistencies did not reap any more rewards. The programme lagged behind the discovery of 'facts'" (154). That is, Lakatos denigrates the change on the basis of it not ultimately resulting in novel success, which, as we saw above, for Lakatos amounts to the degeneration of a research programme. Yet, once again, such retrospective justification seemed not to be required for contemporary physicists to view the

\footnotetext{
${ }^{22}$ Non-relativistic quantum mechanics turned out to be a limit of Dirac's relativistic equation of the electron.

${ }^{23}$ Lakatos appears to suggest that Bohr himself already had that modification in mind when designing the original model (thanks to Helge Kragh for pointing this out to me). There is no textual evidence that Bohr did, but in any case, this seems to matter not so much for whether or not the original Bohr theory, or programme, bore that suggestion.
} 
Sommerfeld model favorably. Bohr, for example, in an attached note to Uhlenbeck and Goudsmit's Nature article in 1926, wrote that the concept of spin "promises to be a very welcome supplement to our ideas of atomic structure" (264). Around the same time, in a letter to Kronig, Bohr described his ultimate conversion to Uhlenbeck and Goudsmit's idea: "I have never since faltered in my conviction that we at last were at the end of our sorrows" (Pais 1991, 243). ${ }^{24}$ Also Pauli, who had earlier called spin an "Irrlehre" (mistaken idea), had conceded that "although at first I strongly doubted the correctness of this idea because of its classical mechanical character, I was finally converted to it by [Llewellyn H.] Thomas's calculations on the magnitude of doublet splitting..." (Pauli's Nobel Prize lecture, Dec 13, 1946). Indeed, despite the aforementioned absurd consequence of electron surfaces spinning faster than the speed of light, Pauli was happy to declare in a letter to Bohr on 12 March 1926 that "Now nothing else is left for me than to surrender completely!" (Pauli 1979, 310). Although also Heisenberg was initially skeptical towards spin and sought to accommodate the hydrogen fine structure with his rump model (see above), he ultimately admitted that "naturally, it is an enormous advance that your theory harmoniously explains away all sorts of things like Zwang and the rest" (Heisenberg to Goudsmit, 16 December 1925, cited by (Serwer 1977, 251)). Again, these reactions were anything but negative, regardless of any possible future novel success resulting from the introduction of spin. Indeed, soon after the introduction of spin, the history of the old quantum mechanics came to an end and classical concepts were given up entirely.

\subsection{The Bohr-Sommerfeld model concluded}

Many changes of the Bohr-Sommerfeld model were made in order to accommodate anomalies. According to the standard accounts of novel success, that makes these changes $a d$ hoc. Not so on McMullin's account. Changes to the model in response to anomalies are not ad hoc so long as they are warranted by de-idealisations of the model. As it turns out though, not even McMullin's preferred example supports this idea. Sommerfeld was happy to keep circular motion in his model and even made it the most likely motion in order to accommodate line intensities. There is also no indication in the publications of neither Bohr nor Sommerfeld that they considered going elliptic as some kind of physically necessitated de-idealisation. Bohr, for example, toyed with the idea of introducing elliptic orbits only when trying to accommodate the fine structure of the spectral lines of hydrogen (cf. Section 4.2). Although it is hard to see that de-idealisation could justify the changes of the Bohr model, there seems to be something about the Bohr model that allows for non-ad hoc changes in order to accommodate new evidence. This is by no means guaranteed. Sometimes theories can accommodate new evidence only in an ad hoc fashion. To see this, let us consider briefly

\footnotetext{
${ }^{24}$ Bohr's conversion happened after he learned from Einstein (through Ehrenfest) that the right application of the theory of special relativity could produce the magnetic field needed for electron spin. See ibid.
} 
another historical example, namely the specific heat anomaly that challenged the kinetic theory of heat in the second half of the $20^{\text {th }}$ century. ${ }^{25}$.

\section{An objectionable change}

The kinetic theory of gases conceives of heat as molecular motion. In its most primitive form, as proposed by the German physicist Clausius in 1857, gases consist of tiny, perfectly elastic particles, whose size is negligible. Insisting on the equipartition of energy among all degrees of freedom of the molecules, Maxwell realized that the theory was contradicted by experiment. The theoretically derived expression for the specific heats of gases, $\gamma=\frac{c_{p}}{c_{v}}=\frac{2+n}{n}$ (whereby $c_{p}$ and $c_{v}=$ specific heat capacity at constant pressure, and volume, respectively, and $n=$ the number of degrees of freedom) predicts $\gamma=\frac{5}{3}=1.66$ for point masses with $n=3$. However, measurements for the specific heat of oxygen, nitrogen, and hydrogen at room temperature gave $\gamma=1.42$. Maxwell concluded radically that this "overturns the whole hypothesis [of the kinetic theory of gases], however satisfactory the other results may be" (Maxwell 1860 cited Brush 1976, 194). Around the same time, however, chemists and physicists had realized that most elements are diatomic in the gaseous state. Diatomic molecules, however, would add another two degrees of freedom, namely two rotational degrees of freedom. Furthermore, it was thought that the known spectral line emission data for various gases suggested that gas molecules had to possess some kind of internal, periodic, vibration. But an additional, vibratory degree of freedom, which Maxwell considered in 1875, would not result in the right prediction either: the three translational degrees of freedom plus two rotational and one vibratory degree of freedom yielded $\gamma=\frac{8}{6}=$ 1.33. In a rather ad hoc fashion, Boltzmann, in December 1976, proposed giving up on the vibratory degree of freedom so that $\gamma=\frac{7}{5}=1.4$, in accordance with the measurements. In March of the same year Kundt and Warburg had discovered that mercury vapor had a specific heat of 1.66: a remarkable confirmation of the simplest version of the kinetic theory. Although Maxwell conceded that Boltzmann's proposal was "in striking agreement with the phenomena of the three groups of gases [i.e. monoatomic, diatomic and polyatomic]" and although he called Boltzmann's idea a "somewhat promising hypothesis", Maxwell strongly objected to it on grounds of it lacking physical plausibility (Maxwell 1860 in Brush 1976, 194). In particular, he objected to Boltzmann's assumption about the negligibility of internal vibrations, when, Maxwell was certain, spectral line emission data required it (ibid.). This sentiment was shared amongst other physicists. For example, Lord Kelvin believed that it was "rigidly demonstrable that repeated mutual impact must gradually convert all the translational energy into the energy of shriller and shriller vibrations of the molecule" (1886, cited in Clark 1976, 85). Lord Rayleigh commented in 1900:

${ }^{25}$ Historical and philosophical discussions can be found in Clark (1976), Brush (1976), Nyhof (1988). See in particular de Regt (1996). 
However great may be the energy required to alter the distance of the two atoms in a diatomic molecule, practical rigidity is never secured, and the kinetic energy of the relative motion in the line of junction is the same as if the tie were of the feeblest. The two atoms, however related, remain two atoms, and the degrees of freedom remain six [not five as in Boltzmann's proposal] in number (cited in ibid.).

Only quantum mechanics would eventually bear out Boltzmann. ${ }^{26}$ Until then, "the specific heats ratio anomaly continued to receive a great deal of attention" (Nyhof 1988, 101).

The contrast between Boltzmann's accommodation of the specific heat anomaly and the changes made to the Bohr model are striking. Although in both cases the model / theory was changed in response to anomalies it was facing, and although in both cases match with the phenomena was established, only in the case of the Bohr model were those changes deemed physically plausible. This is particularly interesting given that (i) the Bohr model was tapping in new physical territory, where accepted physics had reached its limits, and given that (ii) also changes to the Bohr model resulted in apparently absurd consequences as, notably, after the introduction of spin. Again, although physically plausible (or even necessary) de-idealisation does not seem to be the right way of thinking about permitted changes, there does seem to be something about the Bohr model that allows new evidence to be accommodated in a non-ad hoc fashion. In contrast, the kinetic theory, with regards to the specific heat anomaly, lacked this capacity.

\section{Conclusion}

In this paper I critically assessed McMulllin's construal of theoretical fertility. Instead of thinking of fertility in terms of novel success, which has figured so prominently in the realism debate of recent years, McMullin conceives of it in terms of the successful (i.e., nonad hoc) accommodation of anomalies by way of de-idealisation. McMulllin believes that this kind of fertility, by way of predestined motivation and justification of the theoretical changes, gives us strong (and perhaps the strongest) grounds for realism. As I showed in this paper, though, McMullin's preferred example, the Bohr-Sommerfeld model of the atom, does not support this idea. De-idealisation does neither figure on the minds of the scientists making the changes, nor is the idea consistent with the actual development of the model itself. At the same time, both of the standard novel success criteria are violated by the changes. Thus, the changes made to the Bohr-Sommerfeld model during its development come out as methodologically unwarranted and ad hoc on both the de-idealization and the novel success picture. And yet, the changes made to the model were considered to be good ones by the physics community of the time. So, what seems right about McMullin's account is that something about the Bohr model allows the model to be changed in a non-ad hoc fashion; it's just questionable that this 'something' can be described in terms of deidealisation. This became also apparent in comparison with the kinetic theory of gases,

${ }^{26}$ According to quantum mechanics, vibrations become significant only at high temperatures (Nyhof 1988, 99). 
which was not able to accommodate the specific heat anomaly in a non-ad hoc fashion; it lacked this capacity. In conclusion, it seems right to say that the Bohr model was a fertile model (with regards to the changes discussed) and that the kinetic theory ceased to be fertile when it came to the specific heat anomaly: the changes made by Boltzmann to save the phenomena simply could not be motivated by the theory. The foregoing discussion thus shows that there exists such a capacity, and that not all theories possess it at any time. What the capacity to motivate non-ad hoc changes that result in empirical success exactly consists of, is yet an open question. Regardless, the fertility of theories, understood in terms of this capacity, deserves greater attention from philosophers who have been very much obsessed with novel success recently, particularly in the realism debate.

What might help to clarify the capacity is that both novel success and fertility seem to be virtuous because they both ensure the non-ad hocness of the theories in question. And non-ad hocness is clearly an epistemic virtue: theories that accommodate evidence in an ad hoc fashion are widely taken to receive less confirmation than theories accommodating that very same evidence in a non-ad hoc fashion. So ultimately, the virtue of both novel success and fertility appear to reduce to the virtue of non-ad hocness. A future account of either of those should probably seek to shed more light on this virtue.

Of course, the case study of this paper by no means gives us sufficient reason to abandon McMullin's account of fertility in terms of de-idealisation; it might provide a plausible account of other cases. Whether or not it does cannot be determined here. What the present discussion does show, however, is that even cases that might seem to fit McMullin's account very well at first glance, may fail to fit it on closer scrutiny. The historical details thus do matter.

Lastly, some remarks concerning the realism debate are in order. My discussion has shown that the development of the Bohr model, contrary McMullin, does not support McMullin's argument for realism, which has it that the M-fertility of a model or a theory is evidence for the model/theory latching onto reality. Could fertility, understood as the capacity to motivate non-ad hoc changes (and as distinct from de-idealisation), ground an argument for realism? Without a more precise understanding of this capacity, this seems difficult to tell. Perhaps one might argue that a theory being true makes it more likely that a theory accommodates the evidence in a non-ad hoc way. The generality of this question cannot be addressed here. However, with regard to the Bohr model of the atom, realism appears to be in bad standing: despite the fact that the Bohr model made some radically false assumptions that figured centrally (such as well-defined electron orbits), it still turned out to be fertile by way of producing non-ad hoc accommodations. Is this then a victory for empiricism? Not necessarily. Empiricists are content with a theory saving the phenomena; how it does so (e.g. in a unified and simple, or convoluted way), really does not matter to the empiricist. If non-ad hocness were indeed linked to an explanatory virtue such as unifiedness, as some have argued, then that would mean that the empiricists would not attribute any deeper meaning to non-ad hoc explanations. In any case, the most important 
lessons to be drawn then from the discussion of this paper are methodological. A fuller assessment of the implications for realism will have to await further work.

\section{References}

Bohr, N. 1915. On the Series Spectrum of Hydrogen and the Structure of the Atom. Philosophical Magazine 29:332-5.

Brush, S.G. 1976. The Kind of Motion We Call Heat: A History of the Kinetic Theory of Gases in the 19th Century. Amsterdam: North-Holland Pub Company.

Cartwright, N. 1983. How the Laws of Physics Lie. Oxford: Oxford University Press.

Clark, P. 1976. Atomism Versus Theromodynamics. In Method and Appraisal in the Physical Sciences: The Critical Background to Modern Science, 1800-1905, edited by C. Howson. Cambridge: Cambridge University Press.

Curtis, W.E. 1914. Wave-Lengths of Hydrogen Lines and Determination of the Series Constant. Proceedings of the Royal Society of London. Series A 90 (622):605-620.

de Regt, H.W. 1996. Philosophy and the Kinetic Theory of Gases. The British Journal for the Philosophy of Science 47 (1):31-62.

- - - 2001. Spacetime Visualisation and the Intelligibility of Physical Theories. Studies In History and Philosophy of Science Part B: Studies In History and Philosophy of Modern Physics 32 (2):243-265.

Eckert, M., and K. Märker. 2000. Arnold Sommerfeld. Wissenschaftlicher Briefwechsel. Band 1: 1892-1918. Deutsches Museum Verlag für Geschichte der Naturwissenschaften und der Tecknik, Berlin, Diepholz, München.

Forman, P. 1968. The Doublet Riddle and Atomic Physics Circa 1924. Isis:156-174.

Hoyer, U. 1981. Work on Atomic Physics (1912-1917). Vol. 2, Niels Bohr. Collected Works. Amsterdam: North-Holland.

Jammer, M. 1989. The Conceptual Development of Quantum Mechanics: Tomash.

Kragh, H. 1985. The Fine Structure of Hydrogen and the Gross Structure of the Physics Community, 1916-26. Historical Studies in the Physical Sciences:67-125.

- - - 2012. Niels Bohr and the Quantum Atom: The Bohr Model of Atomic Structure 1913-1925: Oxford University Press.

Kuhn, T.S. 1977. Objetivity, Value Judgment, and Theory Choice. In The Essential Tension. Chicago: University of Chicago Press.

Ladyman, J. 1999. Review. A Novel Defense of Scientific Realism. Jarrett Leplin. The British Journal for the Philosophy of Science 50 (1):181-188.

Lakatos, I. 1970. Falsification and the Methodology of Scientific Research Programmes. In Criticism and the Growth of Knowledge, edited by I. Lakatos and A. Musgrave. Cambridge: Cambridge University Press.

- - - . 1978. The Methodology of Scientific Research Programmes: Volume 1: Philosophical Papers. Edited by J. Worrall and G. Currie. Cambridge: Cambridge University Press.

Laudan, L. 1981. A Confutation of Convergent Realism. Philosophy of science:19-49.

Magnus, P., and C. Callender. 2004. Realist Ennui and the Base Rate Fallacy. Philosophy of Science 71 (3):320-338.

McMullin, E. 1968. What Do Physical Models Tell Us? Studies in Logic and the Foundations of Mathematics 52:385-396. 
- - - 1976. The Fertility of Theory and the Unit for Appraisal in Science. In Essays in the Memory of Imre Lakatos, edited by R. S. Cohen. Dordrecht: D. Reidel Publishing Company.

- - - 1982. Values in Science. PSA: Proceedings of the Biennial Meeting of the Philosophy of Science Association Two: Symposia and Invited Papers 3-28.

— - - 1984. A Case for Scientific Realism. In Scientific Realism, edited by J. Leplin. Berkeley: University of California Press.

- - - 1985. Galilean Idealization. Studies in History and Philosophy of Science Part A 16 (3):247-273.

Mehra, J., and H. Rechenberg. 1982. The Historical Development of Quantum Theory. 2 vols. Vol. 1. New York: Springer.

Nolan, D. 1999. Is Fertility Virtuous in Its Own Right? The British Journal for the Philosophy of Science 50 (2):265-282.

Nyhof, J. 1988. Philosophical Objections to the Kinetic Theory. The British journal for the philosophy of science 39 (1):81-109.

Pais, A. 1991. Neils Bohr's Times: In Physics, Philosophy, and Polity. Oxford: Clarendon Press.

Paschen, F. 1916. Bohrs Heliumlinien. Annalen der Physik 355 (16):901-940.

Pauli, W. 2015 Nobel Lecture: Exclusion Principle and Quantum Mechanics. Nobelprize.org. Nobel Media AB 2014, 1946 (accessed 13 Feb 2015 ). Available from http://www.nobelprize.org/nobel prizes/physics/laureates/1945/pauli-lecture.html.

- - - 1979. Wissenschaftlicher Briefwechsel Mit Bohr, Einstein, Heisenberg, Ua, Bd. 1, 19191924. New York: Springer.

Psillos, S. 1999. Scientific Realism: How Science Tracks Truth. London: Routledge.

Robotti, N. 1983. The Spectrum of Z Puppis and the Historical Evolution of Empirical Data. Historical studies in the physical sciences:123-145.

Saatsi, J. forthcoming. On Historical Induction, Old and New. Synthese.

Saatsi, J., and P. Vickers. 2011. Miraculous Success? Inconsistency and Untruth in Kirchhoff's Diffraction Theory. British Journal for the Philosophy of Science 62 (1):29-46.

Scerri, E.R., and J. Worrall. 2001. Prediction and the Periodic Table. Studies in History and Philosophy of Science Part A 32 (3):407-452.

Schindler, S. 2013. Novelty, Coherence, and Mendeleev's Periodic Table Studies in History and Philosophy of Science Part A 45:62-69.

Segall, R. 2008. Fertility and Scientific Realism. The British Journal for the Philosophy of Science 59 (2):237-246.

Serwer, D. 1977. Unmechanischer Zwang: Pauli, Heisenberg, and the Rejection of the Mechanical Atom, 1923-1925. Historical studies in the physical sciences:189-256.

Sommerfeld, A. 1916a. Zur Quantentheorie Der Spektrallinien. Annalen der Physik 356 (17):194.

- - - 1916b. Zur Theorie Der Balmerschen Serie. Sitzungsberichte der Königlich Bayerischen Akademie der Wissenschaften Matematisch-physikalische Klasse:425-500.

- - - . 1923. Atomic Structure and Spectral Lines. Translated by H. Brose. London: Methuen \& Co. Ltd.

Stanford, P.K. 2006. Exceeding Our Grasp: Science, History, and the Problem of Unconceived Alternatives. Oxford: Oxford University Press.

Vickers, P. 2012. Historical Magic in Old Quantum Theory? European Journal for Philosophy of Science 2 (1):1-19. 
- - - 2013. A Confrontation of Convergent Realism. Philosophy of Science 80 (2):189-211.

Worrall, J. 1989a. Fresnel, Poisson and the 'White Spot': The Role of Successful Prediction in Theory-Acceptance. In The Uses of Experiment, edited by D. Gooding, T. Pinch and S. Schaffer. Cambridge: Cambridge University Press.

- - - 1989b. Structural Realism: The Best of Both Worlds? Dialectica 43 (1-2):99-124.

- - - 2002. New Evidence for Old. In In the Scope of Logic, Methodology and Philosophy of Science, edited by P. Gardenfors. Dordrecht: Kluwer 